\title{
DIFERENCIACIÓN ESPACIAL DE LOS NIVELES DE POBREZA EN EL GRAN RESISTENCIA EN LA ACTUALIDAD
}

\author{
Isabel Brest
}

\section{Introducción}

La pobreza es un problema complejo y multidimensional y debido a la intensidad que reviste en nuestros días el desfasaje entre crecimiento económico nacional y calidad de vida en los hogares, su estudio requiere la mayor aproximación estadística y geográfica posible para medirla, caracterizarla y orientar más eficazmente acciones y planes de control y ayuda.

En la Argentina, la historia de la pobreza muestra un significativo vuelco a partir de los cambios en el sistema productivo iniciados en la década del sesenta, especialmente en el transcurso de los últimos quince años, cuando en medio de un progreso material sin precedentes, los índices de pobreza alcanzaron proporciones inusitadas.

La creciente aceleración de los cambios producidos en el sistema capitalista, posibilitó transformaciones socioeconómicas en el ámbito urbano, expresadas en la segregación espacial y exclusión social de la población, conformando los denominados "bolsones de pobreza". La ciudad de Resistencia, junto a las demás localidades que conforman su área metropolitana, no escapó a estos cambios y actualmente constituye uno de los aglomerados urbanos más pobres del país, según los datos relevados por la Encuesta Permanente de Hogares. Esta situación y debido a la expansión que experimentó el Gran Resistencia en los últimos años, hace indispensable la realización de un estudio que permita reconocer espacialmente los diferentes grados de las condiciones socioeconómicas y la pobreza. Es por ello que este estudio pretende contribuir con el análisis de la diferenciación espacial de las condiciones socioeconómicas y establecer los niveles de pobreza tanto en su situación coyuntural como estructural, a partir de los datos del Censo 2001.

\section{Fuentes y metodología}

Para alcanzar los objetivos precedentes, en cuanto a la obtención de los niveles de pobreza se aplicó el IPMV (Índice de Privación de Medios de Vida). El IPMV intenta combinar determinantes de la pobreza relacionados tanto con su carácter coyuntural como con su condicionamiento estructural, mediante la aplicación de un Índice de Capacidad de Subsistencia (ICS) y de un indicador de Condición Habitacional (CH)ํ. En la aplicación de este índice se utilizó la base de microdatos correspondientes al Censo 2001.

\footnotetext{
${ }^{1}$ La discusión de las características y operacionalización del IPMV puede encontrarse en Meichtry et al 2003 y Meichtry y Fantín 2004.
} 
Para el análisis de las condiciones socioeconómicas se ha aplicado el ICS (Índice de Capacidad de Subsistencia), por lo que fue necesario recurrir a las siguientes variables censales simples:

Número de integrantes del hogar

Máximo nivel educativo alcanzado por los perceptores del hogar

Total de perceptores de ingreso en el hogar

Total de hogares

La aplicación del índice $\mathrm{CH}$ (Condición de Hacinamiento) requirió las siguientes variables:

- Número de hogares por tipo de viviendas (Tipo "B", rancho, casilla, etc.)

- Número de cuartos de la vivienda de uso exclusivo

- Número de integrantes del hogar

Para la representación cartográfica, debido al volumen y características de la información manejada se decidió trabajar con el Sistema de Información Geográfica ARC/ VIEW. El marco espacial corresponde al aglomerado urbano Gran Resistencia y la unidad de trabajo son los radios censales del mismo. La unidad estadística considerada son los hogares.

Una vez normalizada la información se procedió a la representación cartográfica del IPMV para determinar la distribución espacial de los niveles de pobreza según los tramos de 0 a 0.24 y de 0 a 0.49 ; es decir muy baja y baja capacidad de los hogares para alcanzar la subsistencia (ver Meichtry y Fantín 2004 y en esta revista).

Asimismo, se trabajó con información correspondiente a variables e indicadores simples extraídos directamente de las tablas publicadas del Censo de Población, Hogares y Viviendas del año 2001, con el objetivo de complementar las conclusiones derivadas de variables complejas y determinar el grado de precariedad de las condiciones sanitarias.

Cabe aclarar que el aglomerado urbano del Gran Resistencia comprende el ejido municipal de Resistencia y la zona portuaria contigua constituida por Barranqueras y Puerto Vilelas a lo que se suma la localidad de Fontana hacia el noroeste (Plano 1).

\section{La pobreza en el Gran Resistencia}

Según los datos relevados por 1NDEC (2005), hasta el primer semestre del año 2005 en los 28 aglomerados más poblados del país, el nivel de pobreza medida en cantidad de personas ha disminuido en más de $10 \%$ en el período $2003-2005^{2}$. De todos modos, casi 9 millones de personas - 8.957.000 - viven en situación de pobreza, es decir el 38,5 \% de la población y $28,4 \%$ del total de hogares que nuclean los 28 distritos urbanos censados (Cuadro 1).

\footnotetext{
${ }^{2}$ Debemos tener en cuenta que esta reducción no significa que las personas en condición de pobreza hayan mejorado sustancialmente su situación, ya que con sólo ganar 5 o 10 pesos más por encima del valor de la canasta básica dejan de ser pobres, aunque su situación socioeconómica no sufra modificaciones significativas.
} 


\section{Cuadro 1}

Hogares y personas por debajo de la línea de Pobreza en Argentina. Año 2005.

\begin{tabular}{|c|c|c|c|c|c|}
\hline Pobreza & $\begin{array}{c}\mathbf{1}^{\circ} \text { semestre } \\
\mathbf{2 0 0 3}^{\mathbf{3}}\end{array}$ & $\begin{array}{c}\mathbf{2}^{\circ} \text { semestre } \\
\mathbf{2 0 0 3}\end{array}$ & $\begin{array}{c}\mathbf{1}^{\circ} \text { semestre } \\
\mathbf{2 0 0 4}\end{array}$ & $\begin{array}{c}\mathbf{2}^{\circ} \text { semestre } \\
\mathbf{2 0 0 4}\end{array}$ & $\begin{array}{c}\mathbf{1}^{\circ} \text { semestre } \\
\mathbf{2 0 0 5}\end{array}$ \\
\hline \% Hogares & 42,7 & 36,5 & 33,5 & 29,8 & 28,4 \\
\% Personas & 54,0 & 47,8 & 44,3 & 40,2 & 38,5 \\
\hline
\end{tabular}

Fuente: Encuesta Permanente de Hogares $1^{\circ}$ semestre 2005. INDEC, 2005

Dentro del ámbito nacional, la región del Nordeste es la más afectada, ya que el 45,3 \% de los hogares -y el 56,3\% de las personas- se encuentran en condición de pobreza, siendo el Gran Resistencia el aglomerado peor posicionado con más de $50 \%$ de los hogares en tal situación, a comienzos del 2005. (Cuadro 2).

\section{Cuadro 2}

Hogares y personas por debajo de la línea de pobreza por regiones del país. Año 2005

\begin{tabular}{|l|c|c|}
\hline & \% Hogares & \%Personas \\
\hline Total 28 aglomerados urbanos & 28,4 & 38,5 \\
Regiones & & \\
Patagónica & 19,6 & 25,5 \\
Pampeana & 24,6 & 33,4 \\
Gran Buenos Aires & 27,4 & 37,7 \\
Cuyo & 29,5 & 37,3 \\
Noroeste & 39,4 & \\
49,4 & & \\
Noreste & $\mathbf{4 5 , 3}$ & $\mathbf{5 6 , 3}$ \\
Formosa & 39,8 & 53,1 \\
Corrientes & 44,7 & 53,5 \\
Posadas & 43,9 & 56,8 \\
Gran Resistencia & 50,0 & 60,3 \\
\hline
\end{tabular}

Fuente: Encuesta Permanente de Hogares $1^{\circ}$ semestre 2005. INDEC, 2005

La intensidad y dimensiones que alcanza la pobreza urbana que acompaña el fuerte proceso de urbanización, nos lleva a considerar algunos aspectos del mismo.

En el ámbito provincial chaqueño se distingue el ritmo de crecimiento de su ciudad cabecera que concentra el $37 \%$ de los habitantes, lo cual demuestra su creciente capacidad de atracción y retención de población. Los datos manifiestan que Resistencia, junto a las

\footnotetext{
${ }^{3}$ No se incluye el aglomerado Gran Santa Fe, cuyo relevamiento no se realizó debido a las inundaciones.
} 
Folia Histórica del Nordeste, $\mathbf{N}^{0} 16$ (Resistencia, 2006) IIGHI, CONICET - IH, UNNE)

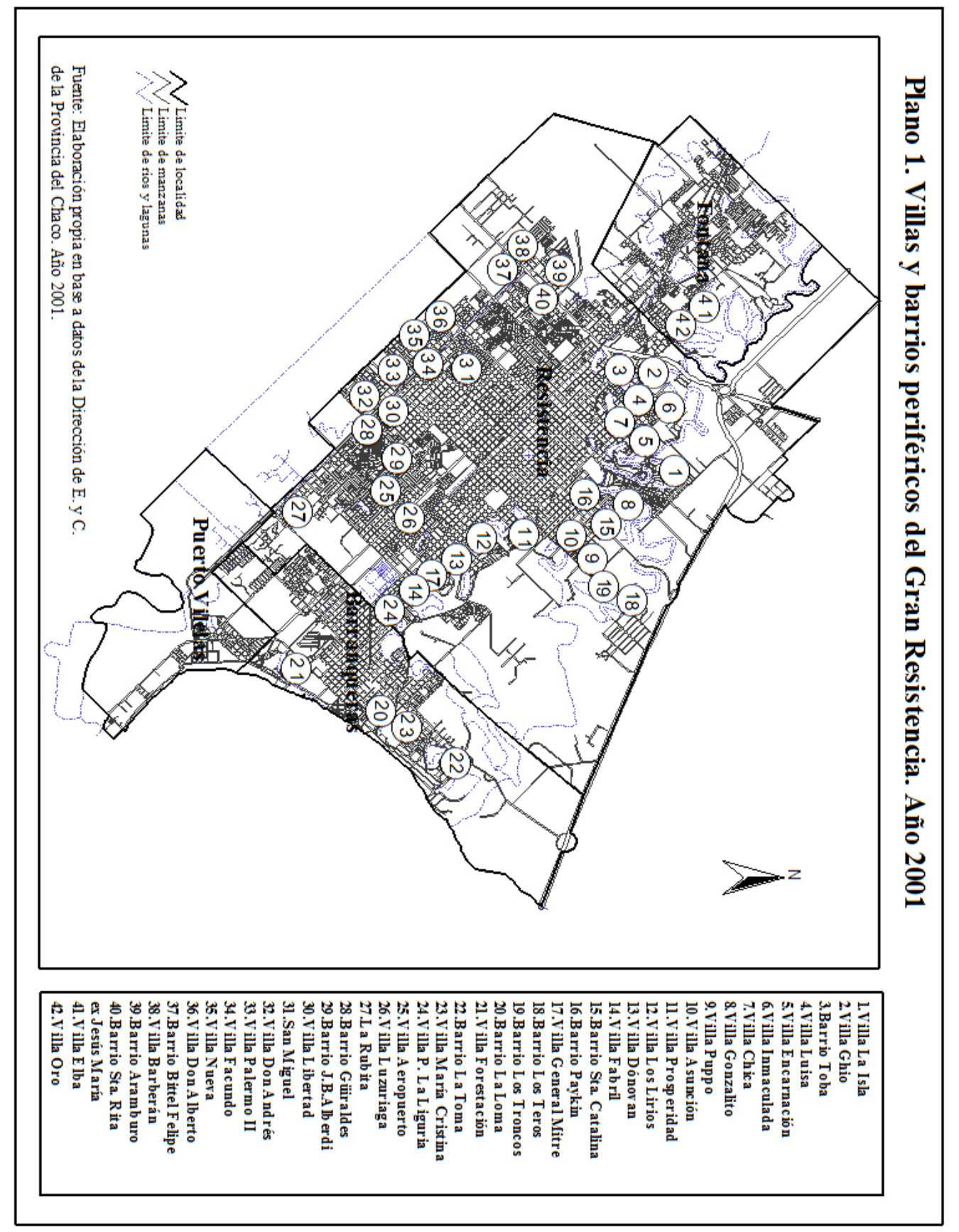


demás localidades que conforman el Área Metropolitana del Gran Resistencia (AMGR) además de constituir la capital de la provincia que muestra elevados índices de carencia, se presenta como una de las ciudades más pobres de todo país y con una población urbana que ha crecido rápidamente en los últimos tiempos. En este sentido, al igual que casi todas las urbes latinoamericanas desde mediados del siglo XX, el AMGR ha crecido no como resultado de un desarrollo armónico sino por el continuo proceso de crecimiento demográfico y por la cada vez mas voluminosa migración rural (Schneider y Alberto, 2005).

Desde la década del ' 70 el proceso de transformación neoliberal, junto a la crisis del monocultivo algodonero ${ }^{4}$ y las sucesivas crisis hídricas locales ${ }^{5}$, generaron en el aspecto espacial la aceleración del proceso irracional de expansión y apropiación del territorio (ocupación "espontánea"), y en el aspecto socioeconómico, nuevas formas de insuficiencia, exclusión y vulnerabilidad para la mayoría de la población ${ }^{6}$.

\section{Distribución espacial de la pobreza. Las situaciones de baja capacidad para alcanzar los medios de vida (IPMV entre 0 y 0.49 ).}

El plano 2 representa el porcentaje de hogares con IPMV entre 0 y $0.49^{7}$, para el año 2001. Dicho intervalo concentra los hogares que se encuentran en situación de baja y muy baja capacidad para alcanzar los medios de vida necesarios y según el ICS -primera parte de la formula- corresponden a hogares que poseen un perceptor de ingresos varón y con nivel de educación secundaria incompleta.

En una primera aproximación en la distribución espacial del índice para el año 2001, debe destacarse que más de la mitad del total de radios censales que componen el Gran Resistencia presentan más del $30 \%$ de sus hogares en condiciones de alta vulnerabilidad ante la pobreza, abarcando importante extensión de la superficie urbana. De este modo, la primera generalización en cuanto a la distribución del fenómeno muestra una condición de fragilidad social espacialmente homogénea.

En general, el IPMV manifiesta espacialmente el cambio gradual desde el centro hacia la periferia; los menores valores se registran en el casco céntrico o próximos a él (menos del 10 $\%$ de los hogares), mientras que los peores niveles de vulnerabilidad se localizan en las zonas periféricas; lo cual pone en evidencia la escasa capacidad de subsistencia de los hogares; pero, al mismo tiempo, está reflejando otras condiciones relacionadas con las diferentes estructuras

\footnotetext{
${ }^{4}$ La competencia de las fibras sintéticas provocó crisis de sobreproducción y, consecuente, caída de los precios.

${ }^{5}$ La alternancia de períodos de inundaciones y sequías, combinadas en muchos ámbitos con el escurrimiento lento del excedente hídrico (Valenzuela de Mari, 1999), significaron importantes pérdidas en la producción agropecuaria, con la consecuente expulsión del trabajador agrario.

${ }^{6}$ La exclusión económica opera a través del mercado de trabajo, restringiendo las posibilidades de las personas de acceder a los medios necesarios para participar en el consumo y producción (específicamente a través del acceso a los factores tierra, capital, capacitación, empleo), situaciones que explican la existencia de hogares con ingresos insuficientes para la subsistencia. Por su parte, la vulnerabilidad ante la pobreza significa la exposición a factores de empobrecimiento y una baja generación de oportunidades para lograr condiciones dignas de vida (Arriagada, 2000).

${ }^{7}$ La escala que representa los porcentajes de hogares se trabajó en cinco cortes naturales establecidos a través del programa ArcView.
} 


\section{Plano 2. Gran Resistencia}

Porcentaje de hogares con IPMV entre 0 y 0.49 por radio censal. Año 2001

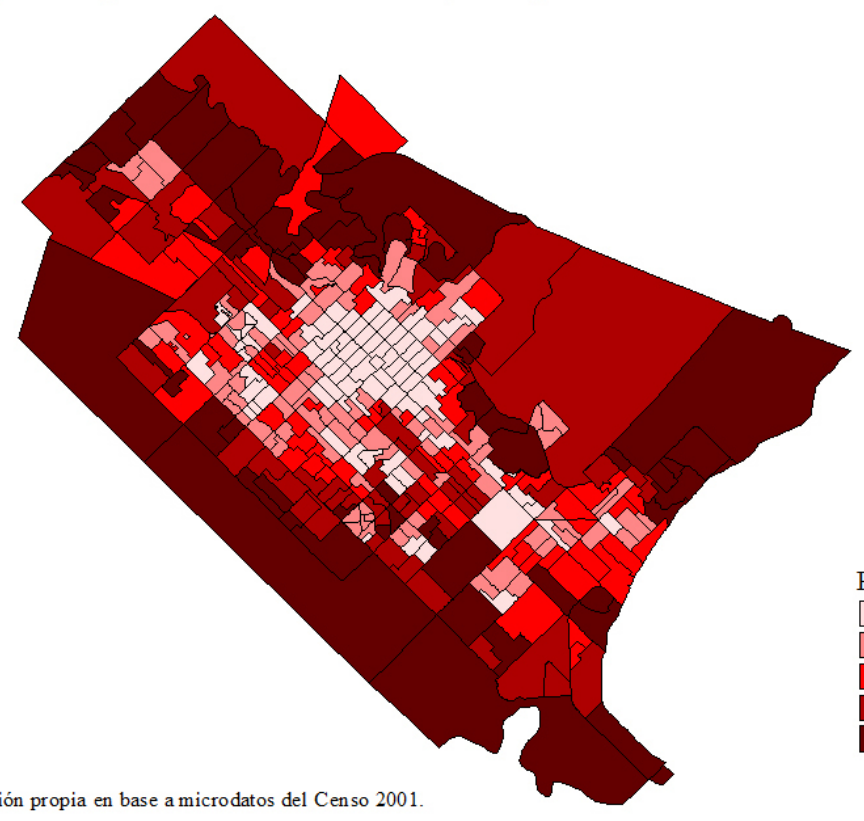

Porcentaje de Hogares $2.75-15.10$

socioeconómicas que -a través de los procesosde expansión urbana- se han intensificado favoreciendo a la zona central del AMGR. Contrariamente, la pervivencia de altos niveles de vulnerabilidad y la desarticulación territorial caracteriza a los cinturones marginales.

En un análisis espacial más detallado, los sectores que poseen la mayor proporción de hogares ubicados en la situación de 0 a 0,49 de IPMV, es decir, baja y muy baja capacidad de subsistencia, corresponden a la periferia urbana, en donde se localizan barrios o villas como Ghio, Luisa, Chica, Elba y Oro hacia el Norte-Noroeste de la ciudad y los barrios La Toma, La Loma, Villa Maria Cristina, General Mitre, Los Lirios y Dónovan al Este. Asimismo, poseen elevado porcentaje de hogares en condiciones de pobreza las villas Don Andrés, Facundo, Alberto, Nueva, Luzuriaga, Aeropuerto y el barrio Bittel Felipe y Juan Bautista Alberdi, entre otros. En estos sectores, los radios censales poseen más del $50 \%$ de los hogares y, en algunos casos, más del $80 \%$ con baja capacidad para obtener recursos necesarios para el sostenimiento.

Por otra parte, se define un círculo intermedio de transición de condiciones de subsistencia heterogénea y menos severa, con valores que oscilan entre 20 y $30 \%$ de hogares con IPMV de 0 a 0.49. 
En la actualidad, las villas que tienen su origen en asentamientos espontáneos conforman en gran medida un cinturón periférico constituido por hogares que padecen de importantes carencias en infraestructura y equipamiento, falta de servicios asistenciales e inseguridad para la adquisición de los terrenos (Mignone, 2001). De este modo, la fuerte deprivación que caracteriza a los habitantes de estos barrios se traduce en niveles críticos de los índices de pobreza.

Asimismo, la mayor proporción de hogares con alta incapacidad de subsistencia - por lo tanto, hogares muy vulnerables- habitan espacios bajos inundables resultantes del proceso de urbanización anárquico y el relleno de antiguos reservorios de desagüe natural (Rey y Olivares, 1997) ${ }^{8}$.

\section{Las situaciones más graves de pobreza (IPMV entre 0 y 0.24 )}

En el plano 3 se ha representado el nivel de privación de medios de vida comprendido entre 0 y 0.24 , es decir el que corresponde a la mayor incapacidad de los hogares en la generación de subsistencia, con el fin de analizar su distribución espacial.

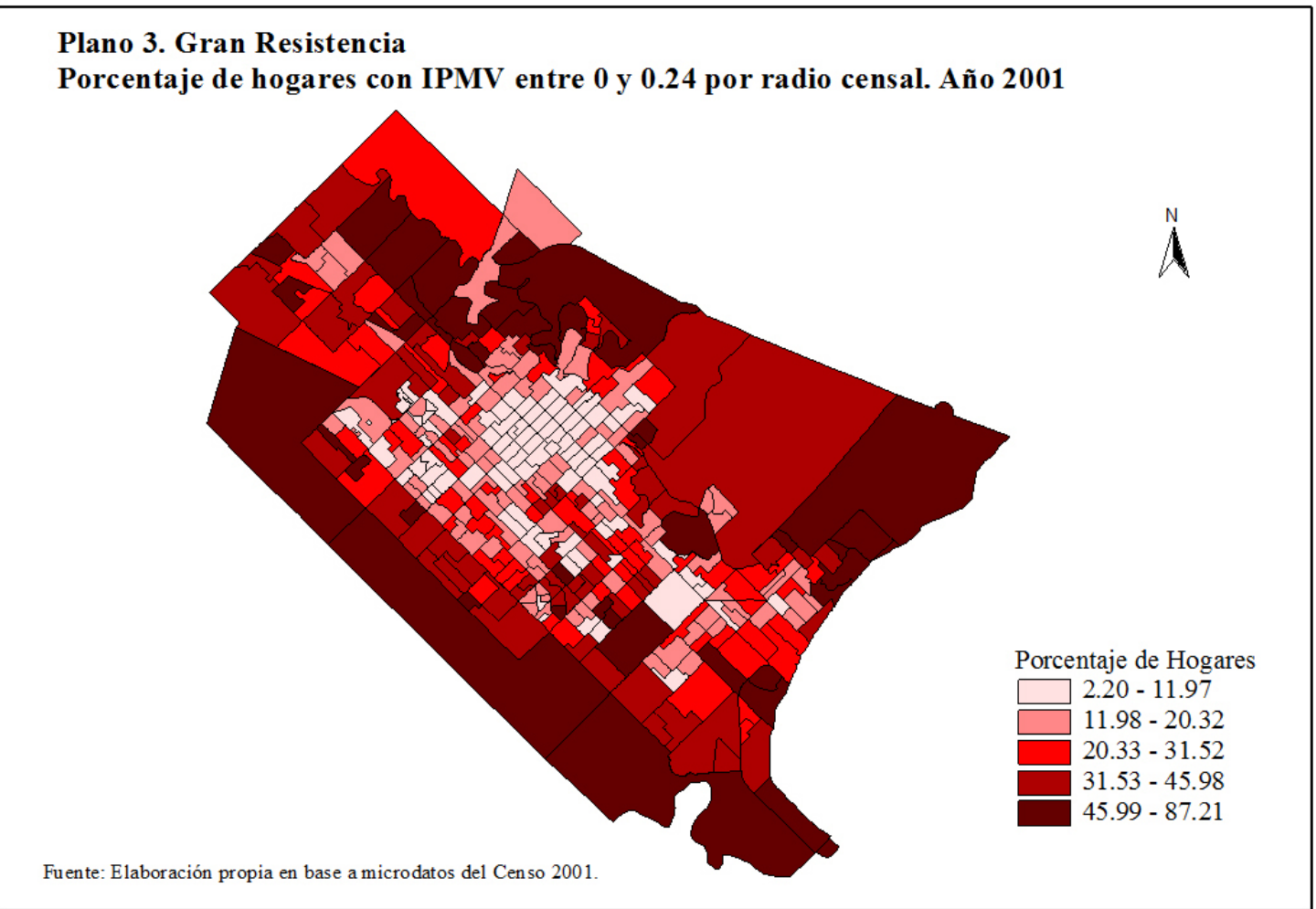

\footnotetext{
${ }^{8}$ Entre algunas villas, se pueden mencionar Villa Maria Cristina, Santo Domingo, Encarnación, Inmaculada y Luisa.
} 
En primer término corresponde destacar la claridad del modelo espacial señalado con anterioridad, al igual que el umbral anterior de 0 a 0.49 , la posibilidad de sostenimiento del hogar es difícil y las familias alcanzan a ser muy pobres en los barrios marginales con porcentajes superiores al $40 \%$ de los hogares con muy baja capacidad de subsistencia. Contrariamente, los que muestran mejores condiciones en la capacidad de generar medios de vida, son los radios ubicados en el centro urbano.

En síntesis, en la estructura urbana de la ciudad de Resistencia existen diferentes niveles y manifestaciones espaciales de pobreza, cuya variación opera fundamentalmente desde el centro hacia la periferia de la ciudad, existiendo un sector espacial intermedio con grupos carenciados de tamaño variable y de localización más dispersa.

\section{Análisis de la distribución espacial de la pobreza según indicadores simples socioeconómicos y de calidad de vida. Año 2001}

Si bien la mayor parte de las variables simples que se han considerado para complementar el análisis de la pobreza están de una u otra manera incluidas en el IPMV; es de interés para este trabajo detectar si la distribución espacial de esas variables con el indicador, determina mayor coincidencia geográfica particularmente con unas que con otras. Así, el análisis espacial por separado de los componentes del IPMV permitirá observar el peso de los mismos.

\section{Nivel de instrucción de la población. Año 2001}

El IPMV comprendido entre 0 y 0.24 detecta las peores condiciones de vulnerabilidad ante la pobreza, ya que los hogares se encuentran en situación de muy baja capacidad para alcanzar medios de subsistencia y según el ICS corresponden a hogares en los cuales el perceptor varón no ha completado el nivel de educación primaria o EGB. Lo cual permite detectar la gravedad que reviste el fenómeno de la pobreza, en cuanto a los recursos necesarios para asegurar la existencia y reproducción del hogar; en especial, cuando se supone que una mayor educación redunda en una inserción laboral mejor rentada.

Según los datos censales del año 2001, el $18 \%$ de la población del Gran Resistencia mayor de 15 años no posee instrucción o no ha completado el nivel primario o EGB, alcanzando valores del $29 \%$ en la localidad de Fontana. Además, existe una gran diferencia a favor de la población que no concluyó el nivel secundario, alrededor del $50 \%$, respecto de las demás categorías (Cuadro 3).

El bajo nivel de instrucción presupone una limitación importante para la participación en el mercado laboral y, por lo tanto, esta situación se relaciona con niveles de ingresos inadecuados o inestables. De allí que la distribución en el espacio urbano de los porcentajes de población mayor de 15 años con educación primaria incompleta (plano 4), marca en términos generales una relación espacial estrecha directa con los índices de IPMV y, constituye una de las insuficiencias más importantes de la población establecida en los márgenes de la ciudad. 


\section{Cuadro 3}

Población de 15 años o más según máximo nivel de instrucción. Año 2001

\begin{tabular}{|l|l|l|c|c|}
\hline Localidades & $\begin{array}{l}\text { Sin instrucción/ } \\
\text { primario o } \\
\text { EGB2 incomp. }\end{array}$ & $\begin{array}{l}\text { Primario o EGB2 } \\
\text { completo/secund. } \\
\text { incompleto }\end{array}$ & $\begin{array}{c}\text { Secundario } \\
\text { completo/ } \\
\text { terciario o } \\
\text { univer. incompl. }\end{array}$ & $\begin{array}{c}\text { Terciario o } \\
\text { universitario } \\
\text { completo }\end{array}$ \\
\hline Gran Resistencia & $\mathbf{1 8 . 4 6}$ & $\mathbf{4 7 . 9 4}$ & $\mathbf{2 5 . 7 7}$ & $\mathbf{7 . 8 4}$ \\
Resistencia & 17.07 & 46.41 & 27.51 & 9.02 \\
Barranqueras & 20.29 & 52.31 & 22.81 & 4.58 \\
Fontana & 28.83 & 54.86 & 13.98 & 2.32 \\
Puerto Vilelas & 25.44 & 54.02 & 17.74 & 2.79 \\
\hline
\end{tabular}

Fuente: INDEC, 2001

En este sentido, los sectores urbanos que poseen elevados porcentajes de educación primaria incompleta muestran alta asociación directa con los valores más críticos de vulnerabilidad (planos 2, 3 y 4 ).

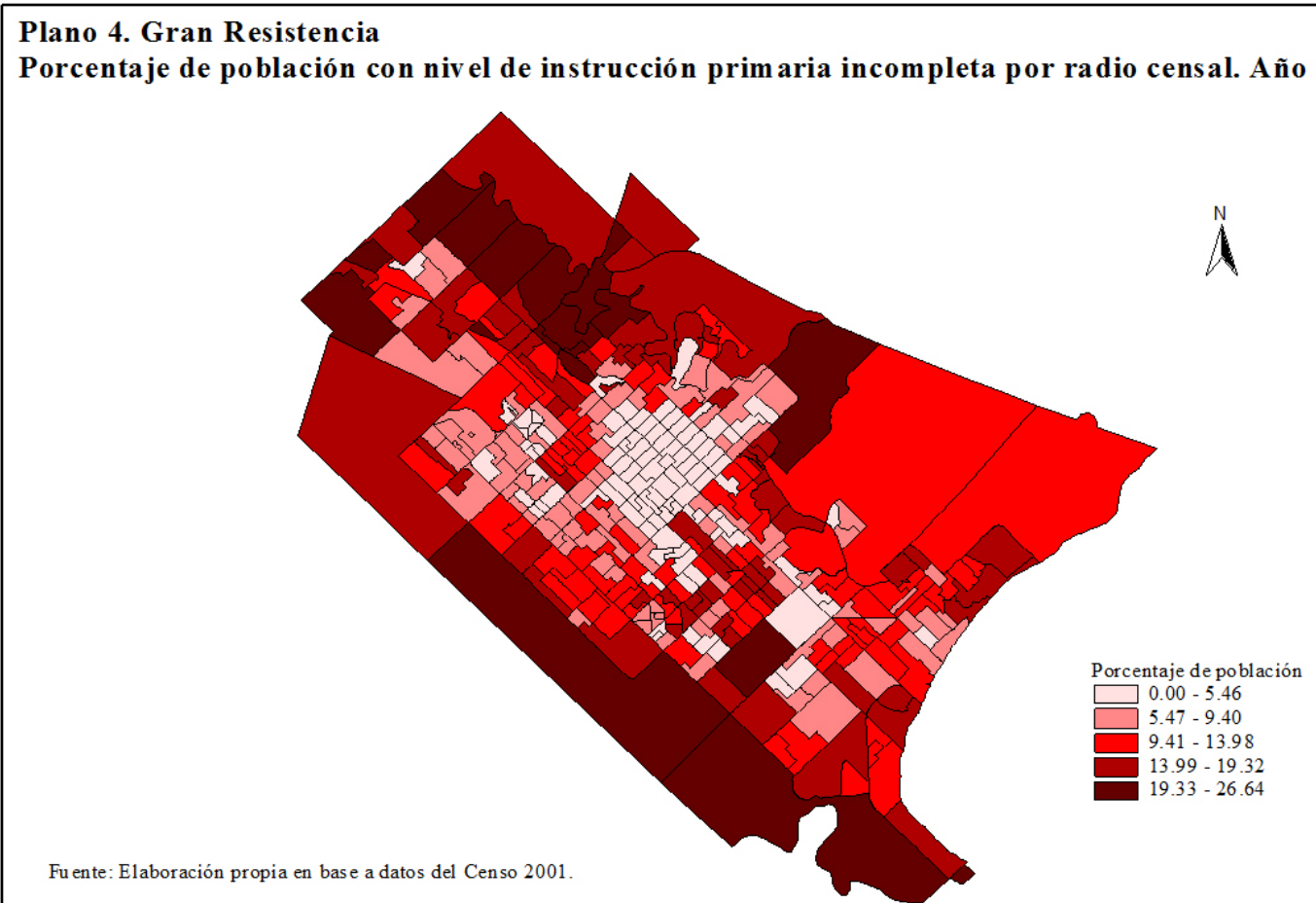




\section{Situación de los hogares según la variable "vivienda deficitaria".}

Según la definición censal, el indicador "tipo de vivienda" se refiere a la situación derivada de la combinación de tres variables: Provisión de agua, características de los servicios sanitarios y el material predominante en los pisos (CEPA, 1993). Por lo tanto este indicador permite analizar la calidad de vida y el grado de vulnerabilidad de la población, ya que la condición habitacional puede ocasionar consecuencias importantes en el bienestar y la salud del hogar. Asimismo, a diferencia de los índices relacionados con la capacidad de subsistencia que expresan la pobreza "coyuntural", las variables relacionadas con la vivienda, son indicativas de la llamada pobreza "estructural".

De acuerdo con el Censo Nacional de Población y Vivienda de 1991, existen diferentes tipos de viviendas particulares; en esta oportunidad se trabajó con las casa tipo B, Rancho y Casilla.

Es importante destacar que el $28 \%$ de los hogares del Gran Resistencia habitan viviendas deficitarias (Cuadro 4) y un tercio de la superficie presenta nivel de carencia crítico; ya que supera el $30 \%$ de hogares en tales condiciones, valor que expresa la magnitud del problema.

En general, la elevada proporción de hogares que poseen viviendas deficitarias corresponden a grupos de familias que han logrado consolidar su vivienda con el correr del tiempo, o tienen cierta estabilidad laboral que les permite invertir en mejoras para la residencia (Mignone, 2001). Pero muchas de ellas, como veremos más adelante, adolecen de falta de retrete con descarga de agua o todavía mantienen el piso de tierra, lo que genera condiciones de alta insalubridad.

\section{Cuadro 4}

\section{Gran Resistencia. Tipo de Vivienda}

\begin{tabular}{|c|c|c|c|c|c|c|}
\hline \multirow{2}{*}{$\begin{array}{l}\text { Hogares } \\
\text { Particulares }\end{array}$} & \multicolumn{3}{|c|}{ Casa } & \multirow{2}{*}{$\begin{array}{l}\text { Rancho o } \\
\text { Casilla }\end{array}$} & \multirow[t]{2}{*}{ Dapartamento } & \multirow[t]{2}{*}{ Ot $\operatorname{ros}^{9}$} \\
\hline & Total & Casa A & Casa B & & & \\
\hline 91.484 & 77.317 & 57.020 & 20.297 & 4.955 & 8.238 & 974 \\
\hline $100 \%$ & $84,51 \%$ & $62,33 \%$ & $22,19 \%$ & $5,42 \%$ & $9,00 \%$ & $1,06 \%$ \\
\hline
\end{tabular}

Fuente: Elaboración propia en base a los datos de Censo de Población y Vivienda 2001.

De acuerdo con el plano 5, la condición habitacional reafirma las disparidades territoriales de los niveles de pobreza. Se advierte la presencia de dos sectores con fuertes diferencias en los porcentajes de viviendas deficitarias y grados de vulnerabilidad. El primero de ellos que posee la mejor condición habitacional, localizado en el centro urbano; el segundo posee más del $40 \%$ de hogares que habitan viviendas deficitarias y constituye el sector más crítico, ya que forma un anillo periférico donde los valores pueden alcanzar más del $90 \%$ de los hogares. Es concordante este modelo espacial con la instalación espontánea y

\footnotetext{
${ }^{9}$ La categoría Otros agrupa casa de inquilinato, hotel o pensión, local no construido para habitar, vivienda móvil y hogares en la calle.
} 
la extensión periférica de sectores de bajos recursos en tierras anegables o de vulnerabilidad hídrica, fiscales o privadas. Además, durante el proceso de urbanización, el tejido urbano del Gran Resistencia fue tomando nuevas formas y dimensiones sin lograr, en el mayor de los casos, condiciones dignas de habitabilidad por carecer de infraestructura, equipamiento adecuado y una efectiva planificación territorial, generando así situaciones de hacinamiento, marginalidad y deterioro de la calidad de vida (Schneider y Alberto, 2005).

\section{Plano 5. Gran Resistencia}

Porcentaje de hogares que habitan viviendas deficitarias por radio censal. Año 2001

Fuente: Elaboración propia en base a microdatos del Cen so 2001

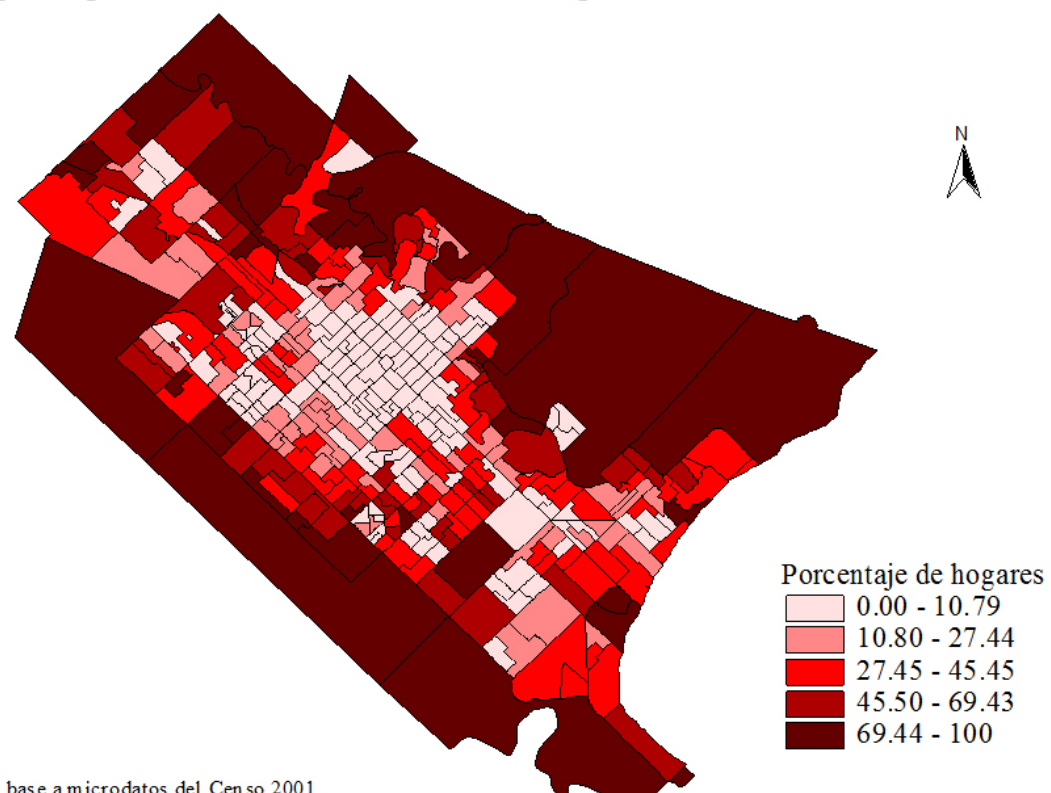

Nuevamente, se detecta un sector intermedio con porcentajes que oscilan entre el 20 y $60 \%$ de viviendas deficitarias, donde aparecen islas de condiciones relativamente buenas. Esta última característica se repite en los barrios planificados y en aquellos equipados en infraestructura de servicios ${ }^{10}$, los cuales mejoran sustancialmente sus índices de pobreza. De este modo su situación habitacional concuerda con las manifestaciones espaciales del índice de privación de medios de vida.

${ }^{10}$ Si la vivienda precaria es uno de los principales indicadores de la pobreza, el déficit y la mala calidad de los servicios públicos constituye también una clara expresión espacial de la existencia de población en condiciones de fragilidad. 
También fue analizado el material predominante en los pisos (plano6) ${ }^{11}$ por su implicancia sanitaria y porque apunta a mostrar el nivel económico de los hogares. En esta ocasión se consideró la categoría piso de tierra, por tratarse de un indicador de las condiciones socioeconómicas altamente desfavorable de los hogares y representar un riesgo para la salud, especialmente de los infantes (Fantín, 1999).

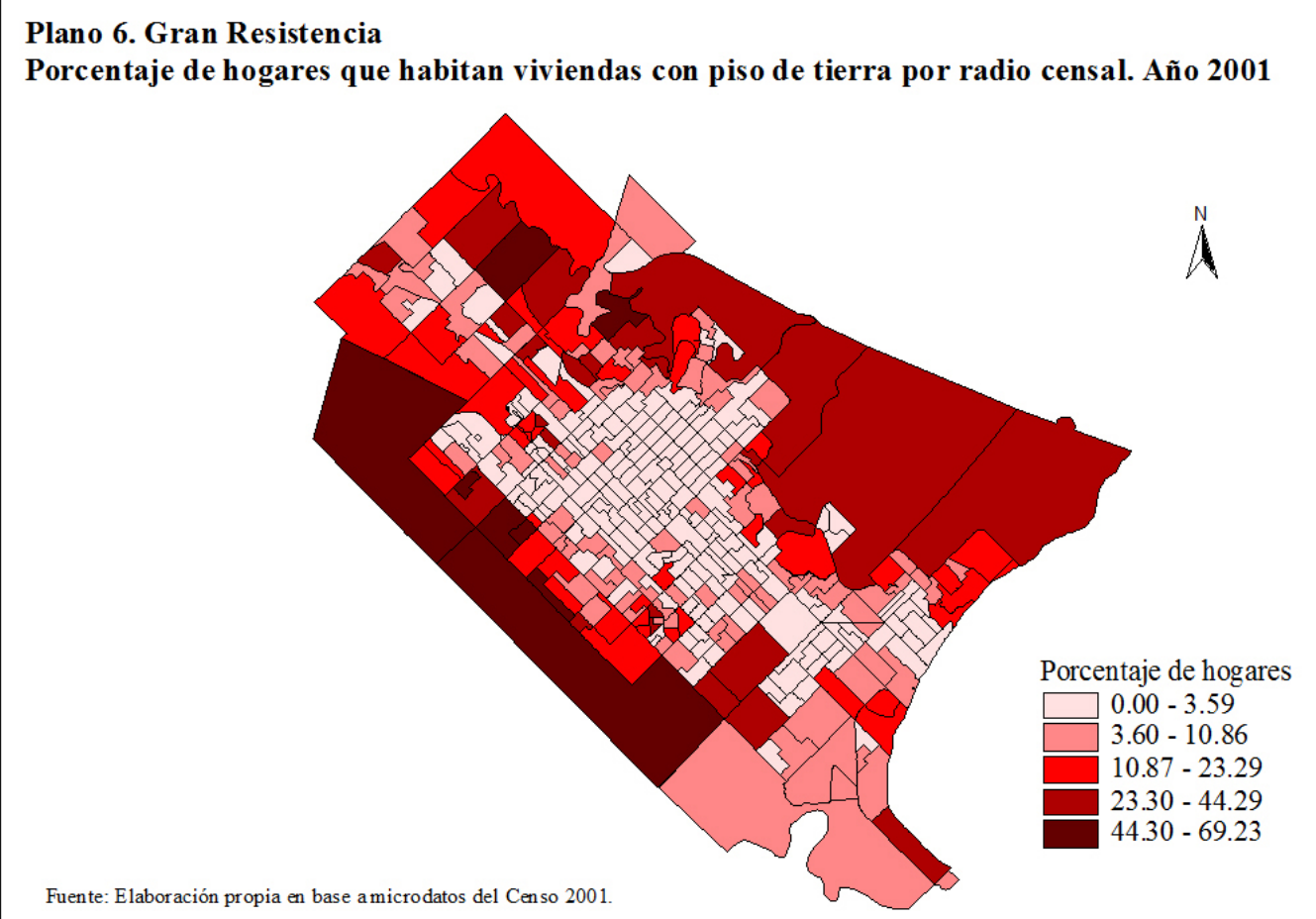

En el plano, se puede observar nuevamente que los mayores porcentajes se encuentran en las áreas periféricas de la ciudad. Asimismo, los valores más críticos de toda la ciudad se localizan en los radios que abarcan las villas Inmaculada, Encarnación, Luisa y Chica al norte de la ciudad; mientras que al este sobresalen las villas Los Lirios y Dónovan. Del mismo modo, en el sector sur-suroeste todos los radios superan el $30 \%$ de hogares que habitan viviendas con piso de tierra, en forma coincidente con la mayor concentración de asentamientos irregulares surgidos durante la década de 1990.

\footnotetext{
${ }^{11}$ En el caso de que coexistieran dos tipos de materiales de igual proporción, el INDEC tiene en cuenta, únicamente, al de mayor calidad.
} 


\section{Condición de los hogares según la variable "viviendas con hacinamiento".}

El nivel de hacinamiento afecta la cotidianeidad de quienes habitan la vivienda. Niveles altos aparecen asociados a obstáculos en torno al descanso, la alimentación, la interacción entre los miembros del hogar, el proceso de socialización de los niños, así como situaciones de promiscuidad y escasa privacidad. En muchas situaciones, el hacinamiento asegura la transmisión de ciertas enfermedades e induce a la generación de accidentes domésticos.

Estudios realizados por el INDEC ponen de manifiesto la estrecha relación entre los niveles de hacinamiento y promiscuidad y los niveles de pobreza de los hogares. En este sentido, la distorsión entre niveles de ingresos y los costos de una vivienda con pautas mínimas de habitabilidad impone a los sectores de menores recursos la generación de estrategias para abaratar costos. Un ejemplo de ellas es la reducción de la capacidad habitacional de la vivienda en relación con el número de miembros de la familia (Fantín, 1999).

De acuerdo con este marco, la situación en el Gran Resistencia demuestra que EL $45,3 \%$ del total de radios censales posee más del $50 \%$ de sus hogares con condiciones de hacinamiento, por lo cual gran parte de los radios, además de poseer viviendas construidas con materiales ligeros, manifiestan precariedad habitacional ${ }^{12}$ (plano 7). Según datos de Censo 2001, más del $20 \%$ de los hogares del Gran Resistencia habitan viviendas con hacinamiento (cuadro 5).

\section{Cuadro 5}

Proporción de hogares hacinados según más de 2 personas por cuarto por localidad. Año 2001

\begin{tabular}{|l|c|c|c|}
\hline Localidades & \multirow{2}{*}{$\begin{array}{c}\text { Total } \\
\text { de hogares }\end{array}$} & \multicolumn{2}{|c|}{ Hogares con hacinamiento } \\
\cline { 3 - 4 } & $\mathbf{8 9 . 9 5 9}$ & $\mathbf{2 1 . 8 4 5}$ & $\mathbf{2 4 , 2 8}$ \\
\hline Gran Resistencia & 69.874 & 15.703 & 22,47 \\
Resistencia & 12.348 & 3.426 & 27,74 \\
Barranqueras & 6.010 & 2.222 & 36,98 \\
Fontana & 1.727 & 588 & 34,06 \\
Puerto Vilelas &
\end{tabular}

Fuente: Elaboración propia en base a datos de INDEC, 2001

12 INDEC considera que existe hacinamiento con un valor de 3 o más individuos por cuarto (CEPA, 1993). Sin embargo, en este trabajo consideramos el umbral de más de 2 personas por cuarto. Este umbral fue establecido para la condición de hacinamiento medida por el índice CH del IPMV bajo la percepción de que, particularmente entre adultos, la cohabitación de tres o mas individuos en un cuarto, significa falta de privacidad y un cierto grado de promiscuidad (Meichtry y Fantín, 2003). 
$\mathrm{Al}$ igual que los planos anteriores ${ }^{13} \mathrm{el} \mathrm{modelo} \mathrm{espacial} \mathrm{se} \mathrm{repite.} \mathrm{Las} \mathrm{áreas} \mathrm{marginales}$ alcanzan a superar el $60 \%$ de los hogares con hacinamiento.

Nuevamente, los sectores sur-suroeste y norte-noroeste son los más afectados y se encuentran ocupados, en muchos casos por villas espontáneas; especialmente los radios que siguen el eje continuo de distribución de las mismas, por el sur hasta el suroeste de la ciudad, a lo largo de las primeras chacras después de la Av. Soberanía Nacional (Mignone, 2000). Asimismo, los radios ubicados hacia el noroeste de la ciudad manifiestan elevados índices y concuerdan con la distribución de hábitat popular.

\section{Plano 7. Gran Resistencia}

Porcentaje de hogares que habitan viviendas con hacinamiento por radio censal. Año 2001

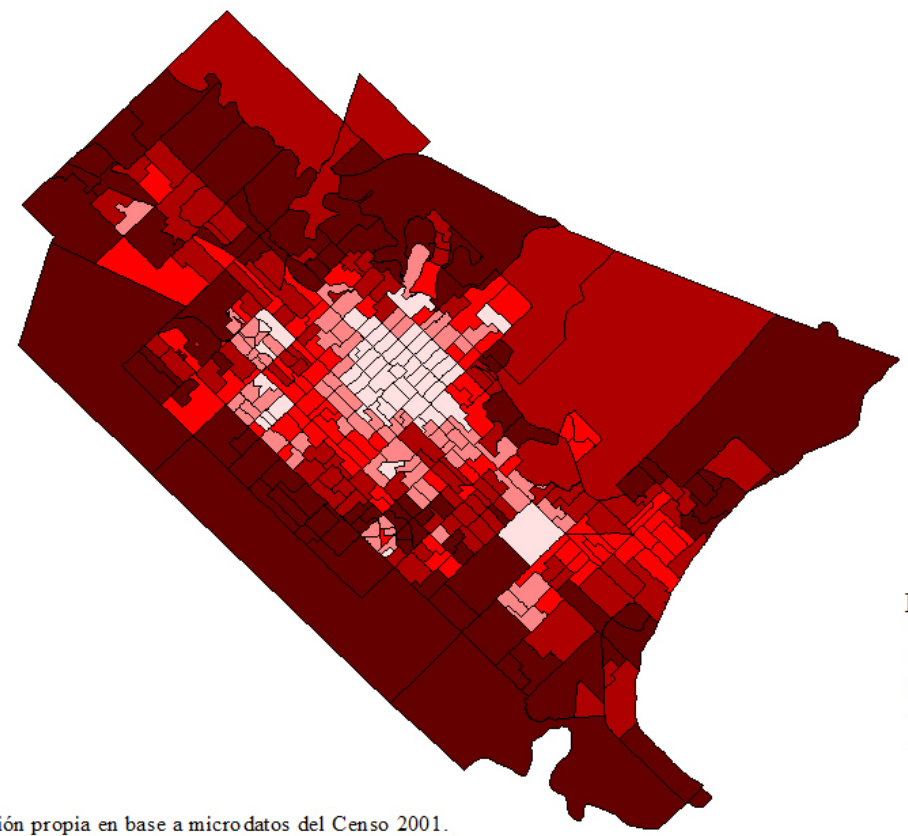

Fracciones y radios.shp

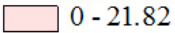

$21.82-35.23$

$35.23-48.98$

$48.98-63.68$

$63.68-86.84$

\section{Provisión de agua y características sanitarias}

Otro de los principales indicadores que merece ser analizado en el estudio de la calidad de vida y la pobreza, por su estrecha relación con la higiene y la salud del hogar, es la calidad del agua en cuanto a la procedencia y la forma de abastecimiento. En este sentido,

${ }^{13}$ La mayor parte de los barrios o villas severamente afectados se localizan en la periferia. Entre ellos se puede mencionar las villas Ghio, Luisa, Chica, Encarnación, Santo Domingo, La Isla, Gonzalito y los barrios Toba, Santa Catalina y Paykinen hacia el Norte; Villa Aeropuerto, Libertad, Don Alberto, Facundo, Palermo II, Don Andrés, el barrio Juan Bautista Alberdi y Bittel Felipe hacia el Sur-Suroeste. Hacia el Este la criticidad aumenta en las villas Los Lirios, Dónovan, Puppo y Asunción. Por último, en el Oeste sobresalen con elevada proporción de viviendas con hacinamiento el barrio Villa Barberán y Aramburu. 
el estudio de la provisión de agua permite establecer futuras relaciones entre hábitat y salud; en particular la relación que existe entre morbi-mortalidad infantil y calidad y acceso al agua.

Según el censo de 1991, aproximadamente el $92 \%$ de los hogares del Gran Resistencia contaban con agua de red y el $70 \%$ poseía conexión dentro de la vivienda (Fantín, 1999). Según los datos del censo 2001, la situación ha mejorado, pues el $98 \%$ de las viviendas tiene acceso al agua corriente y el $80 \%$ posee conexión dentro de la vivienda. El resto de los hogares se abastece por medio de canillas públicas cercanas a su domicilio.

En el plano 8, se observa marcada generalización espacial de niveles bajos. Sin embargo, sobresalen negativamente los radios que agrupan los barrios Los Teros y Los Troncos hacia el norte y los radios periféricos hacia el sur de la ciudad.

\section{Plano 8. Gran Resistencia}

Porcentaje de hogares que habitan viviendas sin agua de red por radio censal. Año 2001

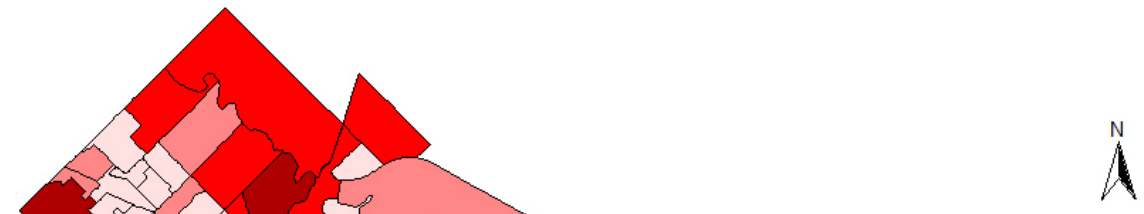

Es importante recordar que en los asentamientos urbanos contar con agua de red es relativamente usual; de todos modos la carencia de este servicio básico depende mayoritariamente de su localización respecto de una red establecida. Implica la existencia de responsabilidad e inversión pública y, al mismo tiempo, se asocia a diferentes niveles de posibilidad económica y preocupación de los hogares en cuanto a su conexión dentro de la vivienda. Por ello, esta variable constituye una de las principales privaciones para la 
evaluación de la dimensión estructural de la pobreza, ya que el acceso a este servicio es considerado imprescindible para el desarrollo de la vida en sociedad.

Estas características se ven reflejadas en el plano 9, donde los radios pertenecientes al área central y próximos a él presentan los porcentajes más bajos de hogares que habitan viviendas sin conexión de agua en su interior; mientras que los terrenos periféricos, muchos de ellos ocupados por asentamientos marginales, presentan los índices de carencia más críticos; es decir más del $30 \%$ de los hogares no cuentan con la instalación de este servicio en su vivienda. La provisión de servicios básicos no ha acompaño al crecimiento de estos sectores de la ciudad. Una necesidad vital como el agua presenta dificultades en su servicio, desde la colocación de cañerías domiciliarias hasta la canilla pública para el asentamiento, o incluso el aprovisionamiento con tanques municipales (Mignone, 2000) ${ }^{14}$.

Entre los radios con elevada proporción se pueden mencionar a aquellos ocupados por La Rubita y los radios contiguos, hacia el sur-sureste; las villas Don Andrés, Palermo II, Facundo, Nueva y Don Alberto al sur-suroeste. Asimismo, las villas Asunción, Prosperidad, General Mitre, Fabril, Los Lirios y Dónovan y un gran número de radios periféricos aislados también poseen elevada proporción de hogares sin conexión de agua dentro de la vivienda.

\section{Plano 9. Gran Resistencia \\ Porcentaje de hogares sin conexión de agua dentro de la vivienda por radio censal. Año 2001}

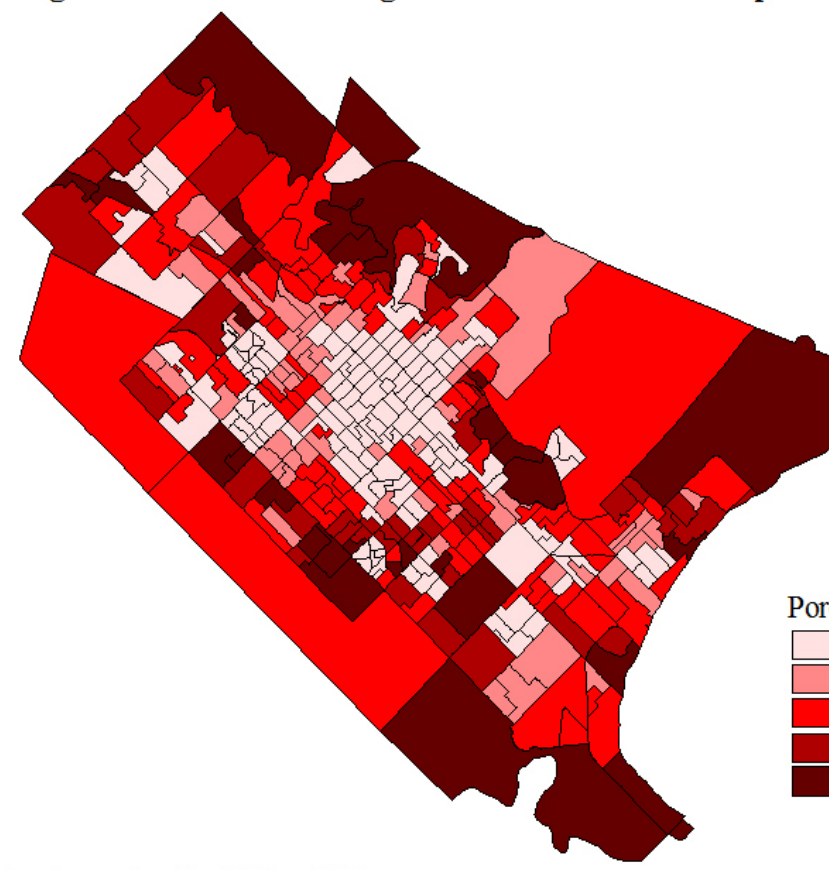

Fuente: Elaboración propia en base a microdatos del Censo 2001.

14 El autor cita el ejemplo correspondiente a las familias del asentamiento "San Miguel", ya que las mismas se abastecen de una sola canilla pública... la que durante el día exhibe una larga cola de amas de casa, niños y jóvenes munidos de baldes, tachos y palanganas (Mignone, 2000). 


\section{Hogares con retrete sin descarga de agua o sin inodoro}

Otro indicador analizado es el porcentaje de hogares con inodoro sin descarga de agua a una red pública ${ }^{15}$ (plano 10) por su relación con el bienestar material, la higiene y por lo tanto con la salud de la familia; pero a diferencia de la calidad del agua, la carencia de un elemento tan básico como el retrete pone en evidencia el déficit de equipamiento de las viviendas y depende más bien de la situación del hogar (Velázquez, 2001).

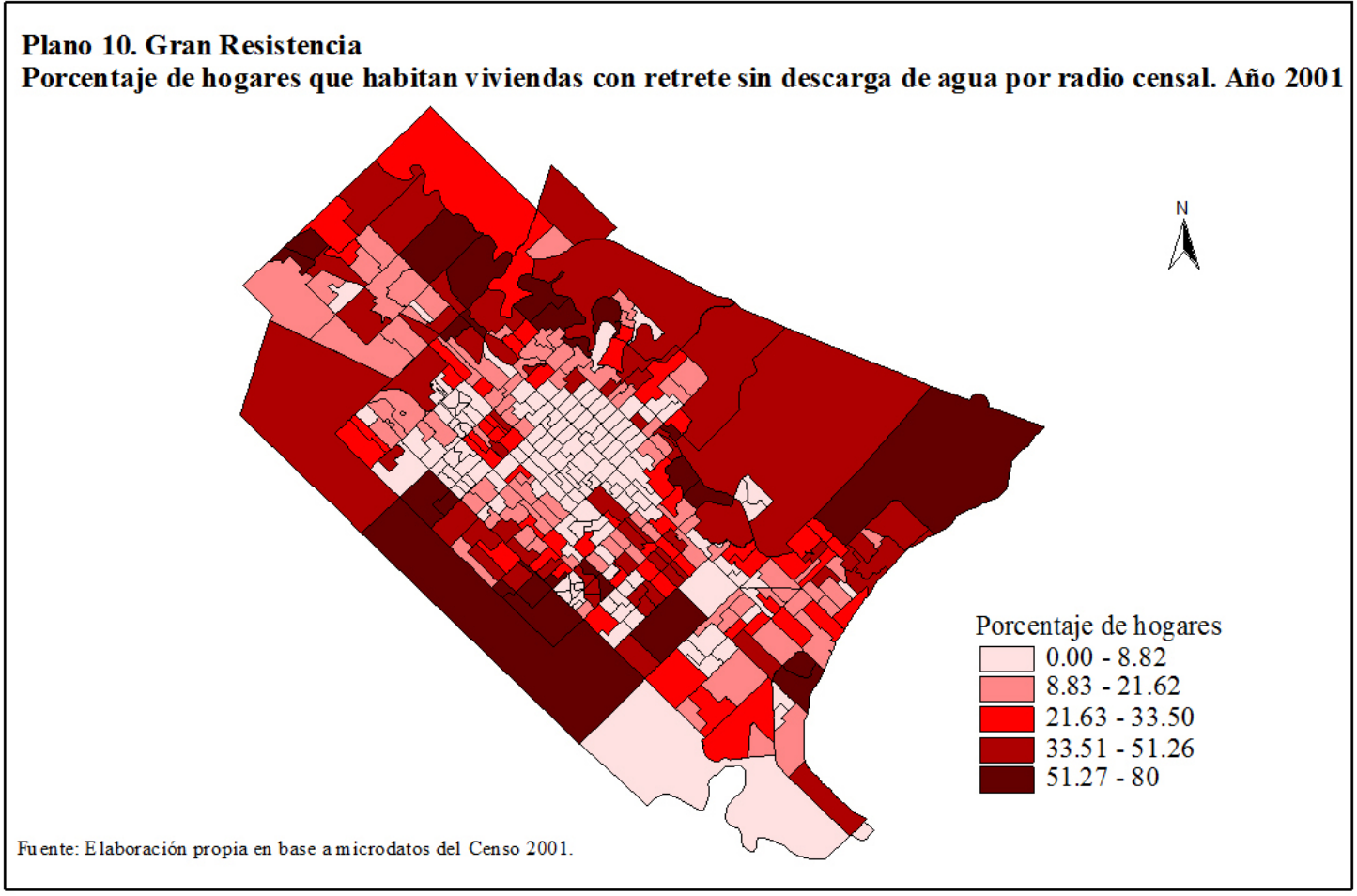

En el año 2001, 20,3 \% de la población argentina no contaba en su vivienda con la instalación de éste equipamiento básico. En cuanto a la provincia del Chaco, la proporción de población que no goza de este servicio supera ampliamente la media nacional, ubicándose entre las jurisdicciones peores posicionadas (49,2\%) (INDEC, 2001).

Si se observa la manifestación espacial de ambos indicadores en el Gran Resistencia (mapa 9 y 10), la tendencia territorial indica una relación geográfica similar. El área central de la ciudad presenta bajos niveles, mientras que, a medida que nos alejamos y avanzamos hacia sectores del borde ejidal, los radios que poseen las mayores deficiencias en cuanto

${ }^{15}$ Se trata de hogares que no poseen inodoro con descarga de agua a una red de cloacas, sino que poseen retrete conectado directamente a un pozo, hoyo o excavación (INDEC, 2001). 
a la procedencia de agua, también disponen de precarias instalaciones sanitarias, es decir más de $50 \%$ de los hogares, especialmente hacia el noroeste y sur de la cuidad.

Nuevamente el cambio gradual de los índices opera desde el centro a la periferia. El modelo se repite y entre ambos sectores se encuentra un sector intermedio ${ }^{16}$ donde los valores definen una transición de marcada heterogeneidad.

Indudablemente, las manifestaciones espaciales más críticas de los dos índices (provisión de agua y presencia o no de inodoro en la vivienda) coinciden; pero con porcentajes mayores en lo que se refiere a los hogares sin inodoros, lo que revela la existencia de casas que tal vez poseen conexión de agua en el interior pero carecen de las adecuadas instalaciones sanitarias; por ejemplo, el sector suroeste que posee entre el 20 y $30 \%$ de viviendas sin conexión de agua; pero más de la mitad de éstas no cuentan con inodoro o retrete con descarga de agua. Los radios que limitan con la ruta nacional Nicolás Avellaneda, desde la ciudad de Barranqueras hasta la intersección con la avenida Sabín, también constituyen ejemplos de estas condiciones.

Asimismo, hacia el noroeste se detectan radios censales que poseen entre 30 y 50 $\%$ de hogares sin inodoro; mientras que los porcentajes de casas sin conexión de agua no constituyen los niveles más críticos del aglomerado urbano.

De todos modos, no debemos olvidar que entre ambas variables existen diferencias primarias: la instalación de agua por red implica la existencia de responsabilidad e inversión pública y, al mismo tiempo, se asocia a diferentes niveles de posibilidad económica y preocupación de los hogares en cuanto a su conexión dentro de la vivienda. En cambio, el retrete implica mayor inversión y depende más bien de la situación individual de la familia.

\section{Consideraciones finales}

La creciente aceleración de los cambios producidos en el sistema capitalista, posibilitó transformaciones socioeconómicas en el ámbito urbano, expresadas en la segregación espacial y exclusión social de la población, conformando los denominados "cinturones de pobreza". El Área Metropolitana del Gran Resistencia, no quedó exenta a estos cambios y, actualmente es el aglomerado urbano que detenta uno de los peores niveles socioeconómicos y de vulnerabilidad ante la pobreza del Nordeste y del conjunto nacional.

En general, la capacidad de subsistencia de los hogares, según la distribución espacial del IPMV de 0 a 0.49 (situación de baja y muy baja capacidad de subsistencia de los hogares), degrada notablemente desde el centro hacia la periferia del Gran Resistencia. En este modelo espacial se encuentran severamente afectados los radios periféricos donde los mayores valores superan el $50 \%$ de los hogares en el año 2001, especialmente al sursuroeste y norte-noroeste de la ciudad. Lo mismo ocurre para el umbral de 0 a 0.24 de IPMV;

\footnotetext{
${ }^{16}$ En especial para la distribución de hogares sin retrete con descarga de agua.
} 
en la representación cartográfica (plano 3) se observa que más del $40 \%$ de los hogares, en los sectores marginales, se encuentran en condición de muy baja capacidad de subsistencia.

La baja capacidad de subsistencia de los hogares ponen en evidencia los peores niveles de vulnerabilidad; pero, al mismo tiempo, está reflejando otras condiciones relacionadas con las diferentes estructuras socioeconómicas que -a través de los procesos de expansión urbana producto la migración rural, por la crisis de la producción agraria- se han intensificado favoreciendo a la zona central del AMGR. Contrariamente, la pervivencia de altos niveles de vulnerabilidad y la desarticulación territorial caracteriza a los cinturones marginales.

En síntesis, en la estructura urbana de la ciudad de Resistencia existen diferentes niveles y manifestaciones espaciales de pobreza. La variación, hacia niveles críticos de los índices, opera desde el centro a la periferia de la ciudad, lo cual reafirma la importancia de la condición central de la misma en la prestación de los servicios en contraposición a la privación que sufren los habitantes que habitan barrios marginales. Esta situación se debe a la incapacidad de subsistencia de las familias y a situaciones estructurales relacionadas con carencias en la infraestructura de la vivienda (sin instalación de agua, sin retrete) o con condiciones de hacinamiento.

Si bien, los índices de pobreza estructural del país manifiestan un descenso para el año 2001, la situación en el Gran Resistencia, aún se presenta severa y con fuertes diferencias espaciales en la mayoría de los indicadores simples.

En cuanto a la dimensión coyuntural de la pobreza, los valores relativos se incrementaron debido al aumento de los pobres extremos, constituidos por aquellos grupos sociales cuyos ingresos, no alcanzan para cubrir el valor mínimo de la canasta básica de bienes y servicios.

\section{Bibliografía}

Altimir, Oscar. 1979. La dimensión de la pobreza urbana en América Latina. Serie Cuadernos de la CEPAL N² ${ }^{\circ}$, Santiago de Chile.

Álvarez, Gustavo, Ariel Lucareli y Silvia Mario. 2001. "La pobreza a partir de los datos censales: nuevos desarrollos basados en la Capacidad Económica de los hogares. Censo Experimental, Pergamino, 1999”. VI Jornadas de Estudios de la Población. AEPA, Neuquén.

Arriagada, Camilo. 2000. Pobreza en América Latina: Nuevos escenarios y desafios de políticas para el hábitat urbano. División de Medio Ambiente y Asentamientos Humanos, Serie: Medio Ambiente y Desarrollo N²7, Santiago de Chile.

Beccaria, Luis y Néstor López. 1997. "El debilitamiento de los mecanismos de integración social”. En Luis Beccaria y Néstor López (comp.). Sin trabajo. Las características del desempleo y sus efectos en la sociedad argentina. UNICEF, Buenos Aires, Losada.

Bruniard, Enrique y Alfredo Bolsi. 1974. El proceso histórico y los caracteres demográficos y socioeconómicos de la ciudad de Resistencia. Folia Histórica del Nordeste $N^{\circ} 1$, Resistencia, Chaco. 
CEPA. Comité Ejecutivo para el Estudio de la Pobreza en la Argentina. 1993. Hogares con necesidades Básicas Insatisfechas (NBI) 1980-1991. Buenos Aires, Secretaria de Programación Económica, Documento de trabajo 3.

Fantín, María Alejandra. 1999. Condiciones socioeconómicas y salud en el Gran Resistencia a comienzos de la década de 1990. Colección Tesis de Maestría. Centro de Estudios Avanzados. Facultad de Ciencias Económicas, Córdoba.

Fantín, María Alejandra. 1999. Diferenciales socioeconómicos y salud en Chaco y Formosa. Folia Histórica del Nordeste, $\mathrm{N}^{\circ} 14$. IIGHI-CONICET, UNNE. Resistencia.

INDEC. 2003. Mapa de Necesidades Básicas Insatisfechas 2001. Aquí se cuenta. Revista Informativa del Censo 2001, n 7, septiembre 2003.

INDEC. 2005. Incidencia de la pobreza y de la indigencia en 28 aglomerados urbanos. Resultados $1^{\circ}$ Semestre de 2005. Instituto Nacional de Estadísticas y Censos, Buenos Aires.

Manoiloff, Raúl O. 2001. El cultivo del algodón en el Chaco entre 1950 y nuestros días, Resistencia, Meana y Meana Chaco.

Meichtry, Norma. 2001. El gran chaco argentino. Territorio, poblamiento y vulnerabilidad social. Folia Histórica del Nordeste, $\mathrm{N}^{\circ} 15$. IIGHI-CONICET, UNNE, Resistencia.

Meichtry, Norma y Alejandra Fantín. 2003. El índice de privación de medios de vida. Reconsideración según tipo de hogar. XXIII Encuentro de Geohistoria Regional. Facultad de Artes, Universidad Nacional de Misiones, Oberá, Misiones.

Meichtry, Norma y Alejandra Fantín. 2001. Condiciones ambientales, procesos de ocupación y desarrollo y calidad de vida en el Nordeste de Argentina. En: G. Velázquez. Geografía, Calidad de vida y fragmentación en la Argentina de los noventa. Análisis regional y departamental utilizando SIG's. Centro de Estudios de Investigaciones Geográficas. U.N.del Centro de la Prov. de Bs. As.Tandil, pp. 114-137.

Meichtry, Norma et al. 2003. El Estudio de la pobreza en el Norte Grande Argentino: consideraciones teóricas y propuesta metodológica. VII Jornadas Argentinas de Estudios de la Población. AEPA. U.N.Tucumán.

Meichtry, Norma y Alejandra Fantín. 2004. Discusiones operacionales acerca del IPMV en la medición de la pobreza en el Norte grande Argentino. Primer Congreso de la Asociación Latino-Americana de Población (ALAP), Caxambú, Brasil. Versión CD-Rom.

Mignone, Marcelo. 2000. Década de 1990: Los asentamientos espontáneos en el crecimiento de Resistencia. XX Encuentro de Geohistoria Regional. Vol 2. Instituto de Investigaciones Geohistóricas-CONICET, Resistencia.

Rey, Walter y Roberto Olivares. 1997. La organización del espacio y algunas consideraciones ambientales de Resistencia y Presidencia Roque Sáenz Peña. Nordeste 5. Segunda época, Facultad de Humanidades, Universidad Nacional del Nordeste, Resistencia.

Schneider, Valeria y Jorge Alfredo Alberto. 2005. Impacto ambiental de la evolución espontánea de ciudades y la capacidad de respuesta estatal. Estudio de caso: evolución del Área Metropolitana del Gran Resistencia. XXV Encuentro de Geohistoria Regional. IIGHI-CONICET, Ministerio de Educación y Cultura de la Provincia de Corrientes, Corrientes.

Rofman, Alejandro. 1999. Desarrollo regional y exclusión social: Transformaciones y crisis en Argentina contemporánea, Buenos Aires, Amorrortu.

Valenzuela de Mari, Cristina. 1991. Los Problemas de la Expansión Urbana y Demográfica de la ciudad de Resistencia, desde la Perspectiva Geográfica a partir de 1960. Cuadernos de Geohistoria Regional No 23. Instituto de Investigaciones Geohistóricas, CONICET, Resistencia. Valenzuela de Mari. Cristina. 1999. Modalidades de expansión en ciudades intermedias argentinas. 
“Urbanización planificada y espontánea en el Gran Resistencia. 1970-1998”. Revista de Geografía Norte Grande No 26, Instituto de Geografía de la Pontificia Universidad Católica de Chile.

Vapñarsky, César A. y Néstor Gorojovsky. 1990. El crecimiento urbano en la Argentina. Instituto Internacional del Medio Ambiente y Desarrollo, Buenos Aires, Grupo Editor Latinoamericano. Velázquez, Guillermo. 2001. Geografía, calidad de vida y fragmentación en la Argentina de los noventa. Análisis regional y departamental utilizando SIGs. Centro de Investigaciones Geográficas. U.N.de Centro de la Prov. de Bs. As., Tandil.

Velázquez, Guillermo. 2001. Factores de diferenciación de calidad de vida en la Argentina. VI Jornadas Argentinas de Estudios de Población. Pp. 635-651.

\title{
Resumen
}

La ciudad de Resistencia, junto a las demás localidades que conforman su área metropolitana, es uno de los aglomerados urbanos más pobre del país, según los datos relevados por la Encuesta Permanente de Hogares.

La representación cartográfica del IPMV (Índice de Privación de Medios de Vida) permite afirmar que en la estructura urbana de la ciudad de Resistencia los niveles de pobreza aumentan desde el centro hacia la periferia, lo cual reafirma los beneficios de habitar en el centro de la ciudad en relación con la mayor la prestación de los servicios, en contraposición a la privación que sufren los habitantes que habitan barrios marginales. Esta situación se relaciona en parte, con la incapacidad de subsistencia de las familias y a situaciones estructurales relacionadas con carencias en la infraestructura de la vivienda ( $\sin$ instalación de agua, sin retrete) o con condiciones de hacinamiento.

$<$ Pobreza urbana $><$ Resistencia $><$ calidad de vida $><$ subsistencia $>$

\begin{abstract}
Resistencia city, together with the centers of its metropolitan area, is one of the poorest urban agglomerates in the whole country, according to the data of the EPH (Continous Households Survey). The cartography of IPMV (Index of Poverty) demonstrates that poverty level increases from downtown to the suburs of the city; that spatial distribution reaffirms the existence of better public services at the central section of the city, in opposition to the almost total absence of them at marginal neighborhoods. That is related to lack of capacity of subsistence of poor families, to structural house deficiencies (without running water, or water closet) and to household heaping conditions.
\end{abstract}

$<$ Urban poverty $><$ Resistencia $><$ quality of life $><$ subsitence $>$ 